Cerebrovasc Dis 2007;23:465

DOI: $10.1159 / 000101752$

\section{The Main Components of Stroke Unit Care: Answer to Dr. Peter Langhorne's Comments}

Didier Leys ${ }^{\mathrm{a}}$, E. Bernd Ringelstein ${ }^{\mathrm{b}}$, Markku Kaste $^{\mathrm{d}}$, Werner Hacke

${ }^{\mathrm{a} U n i v e r s i t y ~ o f ~ L i l l e, ~ L i l l e, ~ F r a n c e ; ~}{ }^{b}$ University of Münster, Münster, and ' University of Heidelberg, Heidelberg, Germany;

dUniversity of Helsinki, Helsinki, Finland

Overall, we agree with Dr. Langhorne's comments on the main components of stroke unit care. By designing this expert survey, we wanted to provide those who are currently implementing stroke units with information on domains that were not covered by properly randomized controlled trials, because they were not part of routine stroke care when trials were conducted.

Besides what has been clearly proven in trials - and was fortunately cited as absolutely necessary by most experts - requirements had to be defined for many other facilities, in the absence of evidence-based data. However, although randomized controlled trials provided clear evidence for the crucial role of several facilities, they should not be overinterpreted for the following reasons:

(i) they were conducted approximately 20 years ago, and many aspects of stroke care had not been evaluated yet at that time (widespread use of MRI, improvements of ultrasonology, monitoring, rt-PA use within $3 \mathrm{~h}$ of ischaemic stroke onset, etc.);

(ii) a need for a more intensive and urgent approach, and, subsequently, the implementation of intensive-care units in many countries;

(iii) stroke patterns may have changed because of the aging of the population and improvements in stroke prevention;

(iv) most trials were conducted in the UK and in Scandinavia, where health systems differ from most of those of the 300 million Europeans who are neither from the UK nor from Scandinavia.

For all these reasons, we think this survey will be useful. Of course, expert opinions do not necessarily make a truth. They have limitations that are discussed in our paper and in Dr. Langhorne's commentary.

Dr. Langhorne is perfectly right when he emphasises that the category 'any hospital ward' is far below the standard level of care.
We just wanted to introduce this possibility because in most European countries, many hospitals still provide stroke care without clearly identified and equipped stroke units. For this reason we wanted to identify the minimum level of care that can be accepted in these hospitals, even if they are organized to refer stroke patients to more equipped units. We will soon present the results of a large European facilities survey that will show that, even if this is a low level of care, many stroke patients in Europe are not even treated in such hospitals.

The term 'stroke centre' used in our survey has little to do with the specific definition and practical transformation of this term in North America. In our paper, it was used as a descriptive term to make the experts aware of the superior level of stroke care.

A misunderstanding is due to the concept of 'comprehensive stroke unit'. 'Comprehensive' means that the hyperacute management is combined with early rehabilitation. For instance, certified stroke units in Germany provide early rehabilitation in the stroke unit itself and continue when the patient is removed from the automated continuous monitoring devices. Comprehensive stroke unit care is routine in Germany and in several other European countries, although it is unlikely that a randomized controlled trial comparing comprehensive stroke centres and conventional care is conducted in the future. Of course, mobile stroke teams cannot replace multidisciplinary comprehensive stroke units.

Differences between 'comprehensive stroke centres' and 'primary stroke centres' are not evidence based. Accordingly, even if there is no evidence-based proof of the benefit of intensive stroke care units, there is indirect evidence that improvements, such as delivery of timely thrombolysis, continuous automated monitoring of vital parameters in the first $72 \mathrm{~h}$, permanent availability of MRI to open the therapeutic time window by mismatch imaging and so on, are truly promising 'add-ons' to the evidence-based standard.

We thank Dr. Langhorne for his interesting comments and his contribution to the exciting and important field of stroke unit care, where not all questions are answered yet.

Prof. Werner Hacke

Department of Neurology, Ruprecht Karl University Heidelberg

Im Neuenheimer Feld 400

DE-69120 Heidelberg (Germany)

Tel. +49 6221568 210, Fax +496221565348

E-Mail Werner_Hacke@med.uni-heidelberg.de 